\title{
Recent Advances in Vaccine Development for the Treatment of Emerging Infectious Diseases
}

\author{
Sahil Kumar', Kiran Thakur², Bandna Sharma², Tilak Raj Bhardwaj², Deo Nandan Prasad³, Rajesh \\ Kumar Singh ${ }^{3, *}$
}

1'Department of Pharmacy, School of Medical and Allied Sciences, GD Goenka University, Gurgaon, Haryana, INDIA.

${ }^{2}$ School of Pharmacy and Emerging Sciences, Baddi University of Emerging Sciences and Technology, Baddi, Himachal Prdesh, INDIA.

${ }^{3}$ Department of Pharmaceutical Chemistry, Shivalik College of Pharmacy, Nangal, Rupnagar, Punjab, INDIA.

\begin{abstract}
Background: Malaria, Chikungunya and Dengue are some of the emerging infectious diseases whose incidence has increased in the past 20 years and could increase in the near future. Although many chemotherapeutic agents are available in clinical use, yet there is a need for the development of novel approaches for the treatment of these lifethreatening diseases. Materials and Methods: In this review, we attempt to highlight some advancement in novel vaccine development in the last decade for the cure of Malaria, Chikungunya and Dengue. The relevant studies published in various reputed journals dealing with different aspects of vaccine development for these diseases are considered only. Results and Conclusion: Many attempts have been made to overcome these infections, but vaccine development for the prophylactic treatment of these infections is still being explored. The present review mainly focused on the vaccines that are under preclinical and clinical stage development and could be an effective weapon in future in the war against these infections.
\end{abstract}

Key words: Vaccine, Malaria, Chikungunya, Dengue, Clinical, Preclinical.

\section{INTRODUCTION}

Malaria is the most fatal human parasitic infection. Malaria is a disease caused by vermin of the genus Plasmodium and it is transmitted via the bites of infected female Anopheles mosquitoes. Four species of Plasmodium, such as Plasmodium falciparum, Plasmodium malariae, Plasmodium ovale and Plasmodium vivax are responsible for causing malaria. The disease is most commonly transmitted by an infected female Anopheles mosquito. The mosquito bite introduces the parasites from the mosquito's saliva into a person's blood. ${ }^{1}$ The parasites travel to the liver where they mature and reproduce. More than 500 million are of malaria yearly if treated in the early stages, malaria can be cured. Five species of Plasmodium can infect and be spread by humans. Most deaths are caused by $P$. falciparum because $P$. vivax, $P$. ovale and $P$. malariae generally cause a milder form of malaria. The species $P$. knowlesi rarely causes disease in humans. For the treatment of malaria, a combination of antimalarial drugs that includes an artemisinin is suggested and the second medication may be either mefloquine, lumefantrine, or sulfadoxine/pyrimethamine. Malaria is a major public health problem and recent control strategies may not be sustainable if drug and insecticide resistance further spread. The burden of disease is mainly concentrated on young children and pregnant women in high endemicity countries in Africa. The first generation malaria vaccine candidate providing partial protection in young children has entered the regulatory pathway for eventual licensure, ambitious goals have been set by the global health community toward the development of second generation high effective vaccines, outlining the need to decrease malaria transmission and protect from Plasmodium vivax
Submission Date: 11-09-2018; Revision Date: 28-12-2018; Accepted Date: 22-04-2019 DOI: 10.5530/ijper.53.3.68 Correspondence:

Dr. Rajesh Kumar Singh, Department of Pharmaceutical Chemistry, Shivalik College of Pharmacy, Nangal, Rupnagar, Punjab- 140126, INDIA.

Phone: +919417513730

E-mail: rksingh244@gmail. com

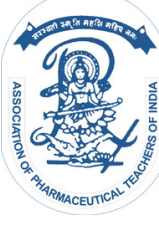

www.ijper.org 
in addition to Plasmodium falciparum. Vaccines providing high short-term protection are within sight. ${ }^{2}$

Chikungunya virus is a growing pathogen initially found in East Africa and presently spread into the Indian Ocean Islands, many regions of South East Asia and in the Americas. No licensed vaccines are available against this eminent pathogen and thus intensive research in this field is a priority. This review presents the recent scenario on the developments of Chikungunya virus vaccines and identifies the use of genetically engineered plants to develop vaccines. ${ }^{3}$ Chikungunya virus (CHIKV) is an arthritogenicalpha virus that during the previous decade has significantly expanded its geographical range and caused large outbreaks of human disease around the world. Although mortality rates related to Chikungunya virus outbreaks are low, acute and chronic illnesses caused by Chikungunya virus (CHIKV) represent a significant load of disease largely affecting low and middle-income countries. This report summarizes the recent status of vaccine development for Chikungunya virus. ${ }^{4}$ Chikungunya virus re-emerged from East Africa to cause devastating epidemics of debilitating and often chronic arthralgia that have affected millions of people in the Indian Ocean Basin and Asia in 2004. More limited epidemics initiated by travelers consequently occurred in Italy and France, as well as human cases exported to most regions of the world, as well as the Americas where Chikungunya virus (CHIKV) could become endemic. Because Chikungunya virus (CHIKV) circulates during epidemics in an urban mosquitohuman cycle, control of transmission relies on mosquito abatement, which is rarely effective. Furthermore, there is no antiviral treatment available for Chikungunya virus infection and no licensed vaccine is available to prevent the disease. Here, we discuss the challenges to the progress of a safe, effective and affordable Chikungunya vaccine and current progress toward this goal. ${ }^{5}$ Chikungunya virus has become a global health problem, spreading to the industrial world of Europe and the Americas; no treatment or prophylactic vaccine is available. Only two candidates have currently qualified to enter clinical phase II trials, a Chikungunya virus-like particle-based vaccine and a recombinant live attenuated measles virus-vectored vaccine. ${ }^{6}$ Chikungunya is an infection caused by the Chikungunya virus. ${ }^{7}$ Symptoms include the fever and joint pain. ${ }^{8}$ These typically occur two to twelve days after exposure. ${ }^{7}$ Other symptoms may include a headache, muscle pain, joint swelling and a rash. The virus is spread between people by two types of mosquitoes: Aedesalbopictus and Aedesaegypti. ${ }^{7}$ They mainly bite during the day. The virus may circulate within a number of animals, including birds and rodents. Diagnosis is by either testing the blood for the virus's RNA or antibodies to the virus. The symptoms can be mistaken for those of Dengue fever and Zika fever.

Dengue fever is a major cause of sickness and death worldwide. The disease is caused by Dengue virus which gets transmitted to humans by the bites of infected mosquitoes, Aedes (Ae.) aegypti and Aedes albopictus. ${ }^{9}$ The disease represents a global health issue as it is endemic in around 100 countries, most of which are in tropical and sub-tropical areas. Over the last decades, the incidence rate and the geographic distribution of Dengue have rapidly increased (Almost 30-fold). Data from the World Health Organization (WHO) estimates up to 390 million cases of Dengue fever each year. ${ }^{10}$ Dengue is a fast growing global health problem. Although various vaccine development challenges exist, numerous candidates are in clinical progress and one has been tested in three clinical endpoint study. The results of the studies have raised numerous questions about how we determine vaccine immunogenicity and how these readouts are related to clinical outcomes in vaccine recipients who experience normal infection. ${ }^{11}$ The present-day candidate vaccines include a liver attenuated tetravalent vaccine, intertype chimeric vaccines based on live attenuated Dengue virus vectors and the chimeric vaccines based on the live attenuated Yellow Fever 17D vector and recombinant vaccines which contain vaccines depend on flavivirus and non-flavivirus vectors. Current studies have shown that the tetravalent formulations may elicit an unbalanced immune response. Research is being carried to the find out means of obtaining a balanced response to all antigens in the tetravalent formulations. ${ }^{12}$ In the present review, we discuss the Dengue vaccine pipeline, growth challenges, the Dengue vaccine-immunologic profiling intersection and research gaps.

\section{Advances in vaccine development for malaria}

\section{Development of Malaria Vaccine RTS, S}

Malaria is spread by Plasmodium falciparum. It is a major public health threat, mainly among children and pregnant women in Africa. Current malaria vaccine candidates are directed against human and mosquito stages of the parasite life cycle, but thus far, for the potential vaccine development, few proteins have been studied. The highly developed vaccine is, RTS, S, conferred partial protection against malaria, in phase II clinical trials and is presently being evaluated in Africa in phase III trial. New vaccine targets need to improve the chances of developing a highly effective malaria vaccine. ${ }^{13}$ 


\section{Vaccines based on the Transmission-Blocking Malaria Vaccine}

Major stumbling blocks include (i) The expression of properly folded target proteins and their downstream purification, (ii) Insufficient induction of sustained functional blocking antibody titers by candidate vaccines in humans and (iii) Validation of a number of (Bio)-assays as compared for blocking activity in the field. Recently, SSM-VIMT candidates, clinical manufacturing and the latest bio-assay development for clinical evaluation has been explored. ${ }^{14}$

\section{Vaccines based on Recombinant Protein}

An advancement in protein vaccine design, development and clinical testing for a number of important malaria antigens from the sporozoite, merozoiteand sexual stages of the parasite's lifecycle-including PfCelTOS, PfMSP1, PfAMA1, PfRH5, Pfs48/45,PfSERA5, PfGLURP,PfMSP 3 and Pfs 25 has been made. Future prospects and challenges for the development, human delivery, production and assessment of protein-based malaria vaccines are discussed. ${ }^{15}$

\section{Development of Malaria Vaccine RTS, S/AS01}

Despite current progress in reducing deaths attributable to malaria, it continues to claim approximately 500,000 lives for every year and is associated with approximately 200 million infections. In 2015, the European Medicines Agency announced the adoption of a positive view for the malaria vaccine candidate most advanced in development, RTS, S/AS01, which provides modest safety against clinical malaria; in early 2016, WHO recommended large-scale pilot implementations of RTS, S in settings of moderate-to-high malaria transmission. ${ }^{16}$

\section{Vaccines based on Whole Organism Blood Stage}

Despite decades of research, an effective malaria vaccine remains elusive. This is due to a number of factors, including: the complex nature and life-cycle of the malaria parasite; its ability to rapidly evolve and evade the host immune system; and the lack of understanding of what precisely mediates immunity. Numerous subunit and whole parasite vaccine approaches targeting different parasite life-cycle stages are being examined in the pursuit of an effective vaccine. Disappointing results following the efficacy testing of sub-unit vaccines in clinical trials, including the most advanced vaccine candidate, RTS, S have highlighted some of the intrinsic limitations of sub-unit vaccines that need to be addressed. Conversely, recent promising results from the clinical testing of radiation attenuated sporozoites have re-ignited interest in the whole parasite approach.
A radiation attenuated sporozoite vaccine must be $100 \%$ effective as even one mosquito-injected sporozoite developing through to blood stage could result in a fulminant blood stage infection. For a whole parasite blood stage vaccine, even a partially effective vaccine could result in lower parasitemias and absence of symptomatic disease in recipients. This review will focus on recent advances in strategies to develop a whole parasite blood stage vaccine. It will also discuss some of the major challenges. ${ }^{17}$

\section{Vaccine based on Development of Sporozoite for Malaria}

Many candidate sporozoite vaccines have been developed and tested by genetic engineering techniques and by polypeptide synthesis. The immunity produced by these synthetic vaccines is mediated by antibody. The human volunteer achieved antibody levels and tests were similar to those resulting from natural infection. Immunity resulting from immunization with irradiated sporozoites is multifactorial. It has cell-mediated components and includes reaction to malaria antigens on infected hepatocytes and such reactions are not induced by synthetic vaccines. Genetic restriction of cell-mediated immune responses may also limit the utility of synthetic vaccines. ${ }^{18}$

\section{Vaccine based on Protein Particle against Malaria}

A recombinant Hepatitis $B$ virus surface antigen (HBsAg) particle vaccine has been developed and showed highly immunogenicity in animal studies and also in human phase I trials and six out of seven human volunteers were protected against sporozoite, the most cheering result of any pre-erythrocytic malaria vaccine. ${ }^{19}$

\section{Cost-Effectiveness of Malaria Vaccine RTS, S/ AS01 in ENDEMIC AFRICA}

RTS, S/AS01 is a safe and more efficacious vaccine considered for implementation in endemic Africa. Model predictions of impact and cost-effectiveness of this new intervention could help in country adoption decisions. The impact of RTS, $\mathrm{S}$ was assessed in 43 countries using an assembly of models of $P$. falciparum epidemiology. Informed by the 32 months follow-up records from the phase 3 trial, vaccine effectiveness was evaluated at country levels of malaria parasite prevalence, coverage of control interventions and immunization. Benefits and costs of the program incremental to regular malaria control were evaluated for a four dose schedule: first dose administered at six months, second and third - before 9 months and fourth dose at 27 months of age. When implemented in all 43 countries the vaccine has the possible to prevent $123(117 ; 129)$ million 
malaria episodes over the first 10 years. Weight averted averages 18,413 DALYs per 100,000 fully vaccinated children with much variation across settings primarily determined by differences in transmission intensity. ${ }^{20}$

\section{RTS, S/AS02D Malaria Vaccine in Infants}

The RTS, S/AS02D malaria vaccine is developed for relief throughout the World Health Organization's Expanded Program on Immunization (EPI). They assessed the feasibility of integrating RTS, S/AS02D into a standard EPI agenda for infants. The controlled trial connecting 340 infants in Bagamoyo, Tanzania, at randomly assigned 340 infants to receive three doses of both the RTS, S/AS02D vaccine or the Hepatitis B vaccine at 8,12 and 16 weeks of age. The main objectives were the incidence of serious adverse events during a 9-month surveillance period and a demonstration of non-inferiority of the responses to the EPI vaccines with co-administration of the RTS, S/AS02D vaccine, as compared with the Hepatitis $\mathrm{B}$ vaccine. The results showed the non-inferiority of the RTS, S/AS02D vaccine in conditions of antibody responses to EPI antigens. The RTS, S/AS02D vaccine receiving $988.6 \%$ of infants after one-month vaccination. The third dose of vaccine. In the 6-month period, the effectiveness of the RTS, S/AS02D vaccine against the first infection with $P$. falciparum malaria was $65.2 \%{ }^{21}$

\section{The Recent Development of a Blood-Stage Malaria Vaccine}

Malaria is the most significant global health problem, responsible for up to 1 million deaths each year. Main efforts have been made to develop an effective vaccine against this disease, to decrease the associated morbidity and mortality. There has already been substantial progress, with the first vaccine against the pre-erythrocytic stages of P. falciparum now a way to licensure. Here, recent progress in the clinical development of bloodstage vaccines is reviewed, as well as methods of antigen selection, the limitations of in vitro assays for selecting vaccines for clinical development and the results of currently published clinical trials. ${ }^{22}$

\section{RTS, S/AS01 Malaria Vaccine in Phase 3 Trial}

In phase 3 trial RTS, S/AS01 malaria vaccine shows $50 \%$ progress both clinical and severe malaria in children 5 to 17 months of age. They studied infants 6 to 12 weeks of age recruited for the same trial. RTS, S/AS01 vaccine is administered in infants with the first vaccination in combination with the Expanded Program on Immunization (EPI) vaccines in a three-dose monthly timetable. Vaccine efficacy against the first or only event of clinical malaria during the 12 months after vaccina- tion, a co-prime endpoint, was analyzed with the use of Cox regression. Vaccine effect against severe malaria was $26.0 \%$ (95\% CI, -7.4 to 48.6$)$ in the intention to take care of the population and $36.6 \%$ (95\% CI, 4.6 to $57.7)$ in the per-protocol population. Serious adverse actions occurred with a similar amount in the two study groups. After administration of the third dose of RTS, S/AS01, $99.7 \%$ of children were positive for anti-circumsporozoite. ${ }^{23}$

\section{Development of Malaria Vaccines}

The depression and suffering caused worldwide by infection with the malaria parasite, particularly P. falciparum, has been well documented. Although no licensed vaccine against malaria recently exists, progress has accelerated in current years towards the goal of developing one. Although the complexity of the malaria parasite has made the malaria vaccine development process tenuous, advances in science and in the vaccine growth process, as well as increases in funding, are cheering. These advances, coupled with the results of the current clinical trial of the vaccine candidate RTS, S, have added new vigor to the thought that a malaria vaccine is not only possible but credible. ${ }^{24}$

\section{Major Challenges to Malaria Vaccine Development}

A safe and effective malaria vaccine would contribute significantly to the control and prevention of the disease. Although a review of global activity in malaria vaccine development does reflect significant activity, progress has remained slow. This article discusses the recent vaccine candidates, with emphasis on those in the clinic and explains the frequent challenges to making and evaluating malaria vaccines, which have resulted in only a few approaches people adopted and repeatedly evaluated. Against a parasite with more than 5200 genes, the lack of perfect information regarding the nature of protective immunity and absence of reliable surrogates of protection is among the key challenges to a rational evaluation and prioritization of candidate vaccines. Pursuing the recent $\mathrm{R}$ and $\mathrm{D}$ strategies may not result in the availability of a vaccine with characteristics suitable to impact appreciably on disease morbidity in developing countries. Therefore, it is critical that the major challenges to malaria vaccine development be definitely identified and collectively addressed. ${ }^{25}$

\section{Development whole Blood-Stage Malaria Vaccines}

There is an increasing realization of the limitations of recombinant protein-based malaria vaccines. This, attached with a better understanding of the protective immunity to malaria, both in animal models and in naturally expose human populations and experimentally 
infected volunteers, as well as the increased capacity to influence parasites provides new impulsion to check whole blood stage parasite approaches to malaria vaccine development. In this review, previous studies in rodents and primates of entire killed and attenuated blood-stage vaccines and current work on the effect of genetically attenuated parasites on immunity in rodent models of blood stage immunity are discussed. The connection between these findings and what is now known about protective immunity in human populations, especially against the blood stages of the parasite lifecycle is discussed and current findings from human experimental infection are being reviewed. At last, the prospect for and impediments to the development whole blood stage parasites are reviewed. ${ }^{26}$ The various advancement in the development of vaccines for the treatment of malaria is shown in (Figure 1).

Some vaccines developed for the treatment of malaria acting at different stages of parasitic infection which are in different clinical trial phases are shown in (Table 1). ${ }^{27}$

\section{Advances in Vaccine Development for Dengue}

\section{Chimeric Tetravalent Dengue Vaccine Candidate}

Dengue is a major problem all over the world and there are no licensed vaccines available for Dengue. Takeda Vaccines Inc. is developing a live, attenuated Tetravalent Dengue vaccine candidate (TDV) that consists of an attenuated Dengue virus-2 (DENV-2) strain Tetravalent Dengue vaccine candidate (TDV-2) and three chimeric viruses containing the prM and $\mathrm{E}$ protein genes of Dengue viruses-1 (DENV-1), -3 and -4 expressed in the context of the attenuated tetravalent Dengue vaccine candidate-2, genome backbone (TDV-1, TDV-3 and TDV-4, respectively). In addition, Tetravalent Dengue vaccine candidate induced $\mathrm{CD} 8(+) \mathrm{T}$ cell responses to the non-structural NS1, NS3 and NS5 proteins of Dengue viruses. The tetravalent Dengue vaccine candidate has been also shown well tolerated and immunogenic in Phase 2 clinical trial in Dengue endemic countries in adults and children as young as 18 months. In addition, clinical studies are in progress in training for a Phase 3 safety and efficacy study. ${ }^{28}$

\section{Sanofi-Pasteur Dengue Vaccine}

The first approved Dengue vaccine has now licensed in six countries. This live attenuated vaccine shows a silent natural infection in boosting host immunity. A transmission dynamic model incorporating this hypothesis fits current clinical trial data well and predicts that vaccine efficacy depends strongly on the age group vaccinated and local transmission intensity. In moderate transmission settings, positive impacts have been observed, but increased risk of hospitalization with Dengue disease for individuals who are vaccinated when seronegative, yet in high-transmission settings, vaccination benefit both the total population and seronegative recipients. This study can help inform policy-makers evaluating this and other applicant Dengue vaccines. ${ }^{29}$

\section{Tetravalent Dengue Vaccine}

The efforts for the development of the Dengue vaccine focused on the development of tetravalent vaccines. However, a current tetravalent vaccine of Phase

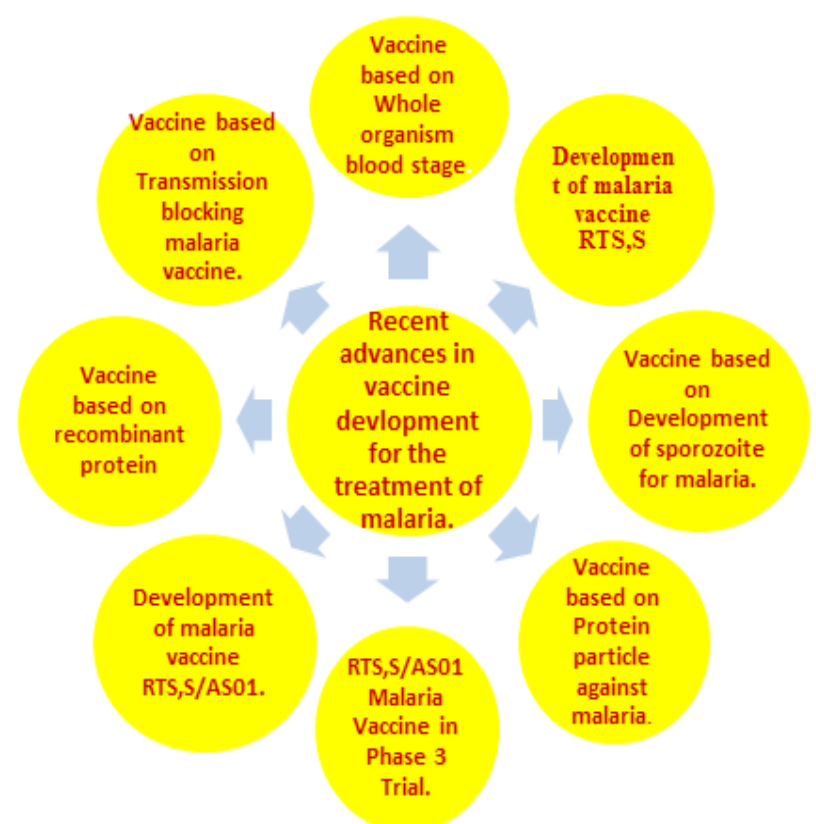

Figure 1: Advances in Vaccine Development for the Treatment of Malaria.

\begin{tabular}{|c|c|c|}
\hline \multicolumn{2}{|c|}{ Table 1: Vaccines for Malaria in Clinical Trial Phase. } \\
\hline $\begin{array}{c}\text { Product name } \\
\text { 257049 }\end{array}$ & $\begin{array}{c}\text { Clinical trial } \\
\text { phase }\end{array}$ & Target stage/site \\
\hline FMP011/AS01B & Phase III & $\begin{array}{c}\text { Pre-erythrocytic } \\
\text { stages }\end{array}$ \\
\hline FMP2.1/AS02A & Phase II I/II trial & $\begin{array}{c}\text { Pre-erythrocytic } \\
\text { stages, Erythrocytic } \\
\text { stages }\end{array}$ \\
\hline FMP1/AS02A & $\begin{array}{c}\text { Pre-erythrocytic } \\
\text { stages, Erythrocytic } \\
\text { stages }\end{array}$ \\
\hline $\begin{array}{c}\text { RTS, S/AS02D } \\
\text { completed }\end{array}$ & $\begin{array}{c}\text { Pre-erythrocytic } \\
\text { stages, Erythrocytic } \\
\text { stages }\end{array}$ \\
\hline $\begin{array}{c}\text { Malaria vaccine } \\
\text { candidates (VAC045) } \\
\text { 1) ChAd63-MVA CS } \\
\text { 2) ChAd63-MVA ME- } \\
\text { TRAP }\end{array}$ & Phase I & $\begin{array}{c}\text { Pre-erythrocytic } \\
\text { stages, Erythrocytic } \\
\text { stages }\end{array}$ \\
\hline
\end{tabular}


IIb trials shows a protective effect against only 3 of the 4 serotypes. Although vaccines effective against a subset of serotypes may decrease morbidity and mortality, particular profiles could result in an increased number of cases due to immune improvement and other peculiarities of Dengue epidemiology. The results show that a partially effective vaccine can have major impacts on serotype distribution and mean age of cases. ${ }^{30}$

Vaccines based on the Chimeric Yellow Fever-Dengue virus (CYD-TDV)

A live-attenuated tetravalent vaccine based on the Chimeric yellow fever- Dengue virus (CYD-TDV) has been developed to phase III efficacy studies. These Dengue vaccine candidates aim to protect against the 4 Dengue serotypes, but the current discovery of a fifth serotype makes difficult vaccine development. ${ }^{31}$

\section{Recombinant Protein Subunit Dengue Vaccines}

Recombinant protein subunit vaccines present a safer alternative to other vaccine approaches. Based on different structural and non-structural proteins of the virus. Several subunit vaccine candidates are presently under development. ${ }^{32}$

\section{Future Trends in the Development of Dengue Vaccines}

Dengue immunity can be elicited via either humoral or cell-mediated routes. Directing the immune response to serotype-specific epitopes from the domain (D)III of the Dengue virus envelope protein (Env) gets effective levels of neutralizing antibodies. Removing serotypecross-reactive epitopes from Domain (D)III, as well as DII, may reduce the potential for the vaccine to induce non-neutralizing antibodies related to Antibodydependent enhancement (ADE) of infection. The use of consensus Envelope DIII sequences for every serotype and maybe even a single consensus Dengue virus sequence for all four serotypes, may direct the immune response to invariant neutralizing sequences, which may improve vaccine safety and long-term efficacy. Vaccines incorporating capsid or Non-structural (NS) proteins may be able to induce tetravalent cell-mediated immunity without antibody-dependent enhancement. However, the potential of cell-mediated immunity to contribute to pathology is not well understood and modifications of non-structural proteins, such as truncated Non-structural (NS1), may be required for optimal vaccine safety. ${ }^{33}$

\section{Development of Next-Generation Dengue Vaccines}

Dengue represents a significant and growing public health problem across the world, just about half of the world's population at risk. The increasing and growing burden of Dengue has highlighted the need for new equipment to prevent Dengue, including the development of Dengue vaccines. Currently, the first Dengue vaccine candidate was evaluated in Phase 3 clinical trials and other vaccine candidates are under clinical trial. There are also a number of candidates in preclinical progress, based on various technologies, with promising results in animal models and probable to move into clinical trials and could finally be next-generation Dengue vaccines. This review discusses the various technological approaches to Dengue vaccine development with specific focus on candidates in preclinical development and with evaluation in non-human primates. ${ }^{34}$

\section{Development of the Sanofi Pasteur Tetravalent Dengue Vaccine}

Sanofi Pasteur has developed a recombinant, live-attenuated, Tetravalent Dengue vaccine (CYD-TDV) specifically in late-stage development. This review summarizes the different steps in the development of Dengue vaccine, with a specific focus on the clinical data from three efficacy trials, which contain some proof-of-concept phase IIb and two pivotal phase III efficacy trials (NCT01373281 and NCT01374516). Previous studies showed that the CYD-TDV candidate had a satisfactory safety profile and was immunogenic across the four vaccine serotypes in both in vitro and in vivo preclinical trials, as well as in initial phase I to phase II clinical trials in both flavivirus-naïve and seropositive individuals. Data from the 25 months (After the first injection) active phase of the two essential phase III efficacy studies shows that CYD-TDV (Administered at 0, 6 and 12 months) is effective against virologically-confirmed disease (Primary endpoint) and has a good safety profile. Secondary analysis also showed efficacy against all four Dengue serotypes and protection against severe disease and hospitalization. Because of the complexities of Dengue disease, both short-term and long-term safety and efficacy need to be addressed by in progress longterm action and future post-licensure studies. ${ }^{35}$

\section{Recent Status in Dengue Vaccine}

An ideal Dengue vaccine must provide long-lasting immunity against all four serotypes of the virus. The envelope protein of the virus plays an important role in vaccine development. The present-day candidate vaccine contains a live attenuated tetravalent vaccine, inter typicchimaeric vaccines based on live attenuated Dengue virus vectors, chimeric vaccines based on the live attenuated Yellow Fever 17D vector and recombinant vaccines which contain vaccines based on flavivirus and non-flavivirus vectors. Tetravalent live attenuated vac- 
cines, intertypic chimeric vaccines and chimeric vaccines are individuals tested in human trials. Recombinant DNA vaccines based on flavivirus and non-flavivirus vectors are individually tested in animal trials. Current studies have shown that the tetravalent formulations may elicit an unstable immune response. Research is continuing to find out means of obtaining a balanced response to all antigens in the tetravalent formulations. ${ }^{12}$

\section{Development of a Live Attenuated Tetravalent Dengue Vaccine}

The development of a live attenuated tetravalent Dengue vaccine is presently the best policy to obtain a vaccine against Dengue viruses. Dengue serotype 1, 2 and 4 viruses were developed in main dog kidney cells, while Dengue serotype 3 was in sequential passages in main African green monkey kidney cells. Tissue culture passaged strain viruses were subjected to biological symbol studies. Candidate vaccines have been tested as Monovalent (Single virus), Bivalent (Two viruses), Trivalent (Three viruses) and Tetravalent (All four serotype viruses) vaccines in Thai volunteers. They were found to be secure and immunogenic in both adults and children. The Mahidol lives attenuated Dengue 2 virus was also tested in American volunteers and resulted in good immune response identical from those induced in Thai volunteers. The master seeds from the four live attenuated virus strains developed were provided to Pasteur Merieux Connaught of France for manufacture on an industrial scale follow good manufacturing practice guidelines (GMP). ${ }^{36}$

\section{Sanofi Pasteur Tetravalent Dengue Vaccine}

Sanofi Pasteur tetravalent Dengue vaccine is composed of four recombinant live attenuated vaccines based on a yellow fever vaccine 17D (YFV 17D) backbone, all expressing the prM and envelope genes of one of the four Dengue virus serotypes. Pre-clinical studies have confirmed that the TV Dengue vaccine is genetically and phenotypically stable, non-hepatotropic, a smaller amount neurovirulent than YFV 17D and does not contaminate mosquitoes by the oral route. In vitro and in vivo preclinical studies also showed that the TV Dengue vaccine-induced controlled stimulation in human dendritic cells and major immune responses in monkeys. TV Dengue vaccine reactogenicity, viraemia induction and antibody responses were investigated in three Phase I trials in the USA, the Philippines and Mexico, in a two or three-dose treatment over a 12 month period. Results showed that the majority of undesirable events were mild to moderate and transient in nature. Viremia was transient and small and was not increased after first Dengue TV administration, even in the case of incomplete responses. ${ }^{37}$

\section{Development on Sanofi Pasteur Dengue Vaccine}

The Sanofi Pasteur tetravalent Dengue vaccine is a recombinant live attenuated vaccine. It is based on a backbone of yellow fever vaccine (YFV 17D) replication genes and integrates the envelope genes of the four Dengue virus serotypes. Pre-clinical studies have confirmed that Dengue TV is genetically stable, nonhepatotropic, a smaller amount of neurovirulent than YF 17D and does not contaminate mosquitoes by the oral route. Dengue TV reactogenicity, viraemia induction and antibody responses were investigated in three Phase II trials in the USA, the Philippines and Mexico. Participants were randomized to be given a three-dose regimen of Dengue TV over 12 months (given at baseline, 3-4 and 12 months) or a control vaccine at baseline followed by two injections of Dengue TV. Results showed that the majority of undesirable events were mild to moderate and transient in nature, whereas no evidence of induction of viraemia was reported after first Dengue TV administration. Seroconversion was $100 \%$ for all four serotypes in flavivirus-naive adults in the USA injected with Dengue TV. In the same way, seroconversion was $88-100 \%$ following three administrations in flavivirus-naive Mexican children age 2-5 years. ${ }^{38}$ The various advancement in the development of vaccines for the treatment of Dengue is shown in (Figure 2).

Some vaccines developed for the treatment of Dengue acting at different stages of parasitic infection which are in different clinical trial phases are shown in (Table 2). ${ }^{12}$

\section{Advances in Vaccine Development for Chikungunya}

\section{A Live Recombinant Measles-Virus-based Chikungunya Vaccine}

The measles-virus-based Chikungunya applicant vaccine is a live attenuated recombinant viral vectored vaccine based on the Schwarz strain of measles vaccine, which was initially introduced by the Pasteur Institute in Paris. Recombinant measles-virus vectors give strong and protecting immunity against many arbo viruses and immunogenic. The immunogenicity and protecting the efficacy of the measles-virus-based Chikungunya vaccine was shown ina measles virus-susceptible mouse model, showing that neutralizing antibodies induced by the vaccine give complete protection against a dangerous challenge with Chikungunya virus. ${ }^{39}$ 


\begin{tabular}{|c|c|c|c|}
\hline Vaccine Type & $\begin{array}{l}\text { Developed } \\
\text { By }\end{array}$ & Country & $\begin{array}{l}\text { Clinical } \\
\text { Trials }\end{array}$ \\
\hline $\begin{array}{l}\text { Live attenuated } \\
\text { tetravalent vaccine }\end{array}$ & $\begin{array}{l}\text { Mahidol } \\
\text { University }\end{array}$ & Thailand & In Phase III \\
\hline $\begin{array}{l}\text { Live attenuated } \\
\text { tetravalent vaccine }\end{array}$ & WRAIR & United States & In Phase II \\
\hline $\begin{array}{l}\text { Intertypicchimaeric } \\
\text { vaccine }\end{array}$ & NIAID, NIH & United States & In Phase II \\
\hline Chimaeric vaccine & Acambis & United States & In Phase II \\
\hline Chimaeric vaccine & CDC & United States & In Phase I \\
\hline $\begin{array}{l}\text { Flavivirus-based } \\
\text { recombinant DNA } \\
\text { vaccine }\end{array}$ & $\begin{array}{l}\text { Navy } \\
\text { Medical }\end{array}$ & United States & In Phase I \\
\hline $\begin{array}{l}\text { Non-flavivirus } \\
\text { based recombinant } \\
\text { DNA vaccine }\end{array}$ & $\begin{array}{l}\text { Hawaii } \\
\text { Biotech }\end{array}$ & United States & Preclinical \\
\hline
\end{tabular}

\section{Recombinant Chikungunya Virus Vaccine Comprising a Non-Replicating Complex Adenovirus}

A recombinant Chikungunya virus vaccine comprising a non-replicating complex adenovirus vector encoding the structural polyprotein cassette of Chikungunya virus (CHIKV) has been developed. A single immunization through this vaccine consistently induced high titres of anti-Chikungunya virus antibodies that neutralized both an old Asian isolate and a Réunion Island isolate from the current epidemic. The vaccine also protected mice against viraemia and arthritic disease caused by both virus isolates. ${ }^{40}$

\section{Novel Consensus-based DNA Vaccine against Chikungunya Virus (CHIKV)}

Novel consensus-based vaccine design for CHIKV, employing a DNA vaccine strategy. The vaccine cassette was designed based on CHIKV capsid and envelope-specific consensus sequences with a number of modifications, including codon optimization, RNA optimization, the addition of a Kozak sequence and a substituted immunoglobulin $\mathrm{E}$ leader sequence. The expression of capsid, envelope E1 and E1 was evaluated using T7-coupled transcription and immunoblot analysis. A newly developed, adaptive constant-current electroporation technique was used to immunize C57BL/6 mice with an intramuscular injection of plasmid coding for the CHIK-Capsid, E1 and E2. Analysis of cellular immune responses, including epitope mapping, demonstrate that electroporation of these constructs induces both potent and broad cellular immunity. In addition, antibody ELISAs demonstrates that these synthetic immunogens are able of inducing high titer antibod-

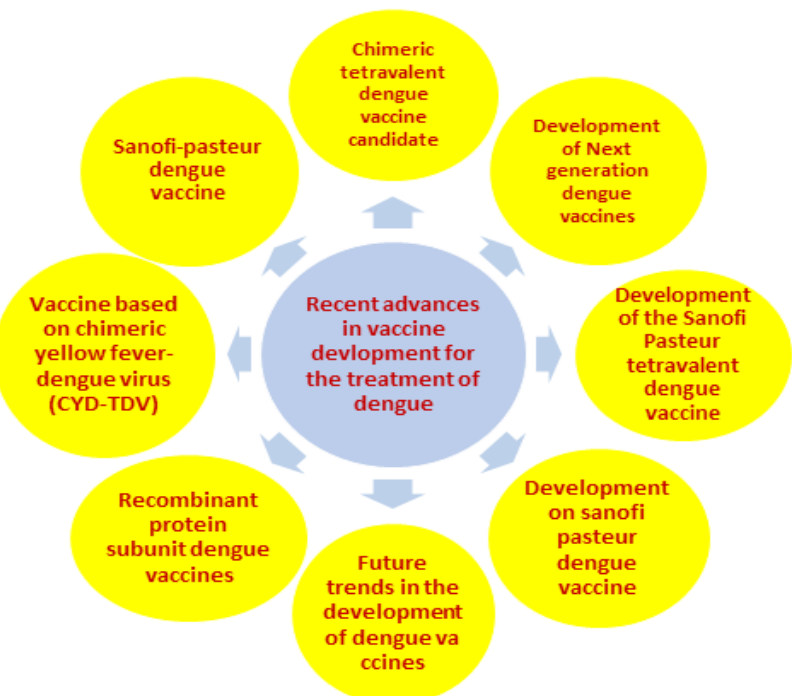

Figure 2: Advances in Vaccine Development for the Treatment of Dengue.

ies capable of recognizing the native antigen. Taken together, these data support further study of the use of consensus CHIK antigens in a probable vaccine cocktail. ${ }^{41}$

\section{Sequencing of DNA for Chikungunya Vaccine}

A novel vaccination strategy based on iDNAs infectious clone designed to launch a live- attenuated Chikungunya virus vaccine from plasmid DNA in vitro or in vivo. As a evidence of concept, iDNA plasmid pCHIKV-7 encoding the full-length cDNA of the 181/25 vaccine has been developed. The DNA-launched Chikungunya virus- 7 was prepared and compared to the $181 / 25$ virus. In the Chikungunya virus (CHIKV-7), frequencies of reversions at E2-12 and E2-82 were $0.064 \%$ and $0.086 \%$, while in the $181 / 25$, frequencies were $0.179 \%$ and $0.133 \%$ respectively. The DNA-launched virus has a reduced probability of reversion mutations, thereby enhancing vaccine protection. ${ }^{42}$

\section{Future Trends in Chikungunya Vaccine Development}

Chikungunya virus is an expanding global public health problem. That needs a global strategy besides vector control which has achieved only limited success in reducing the transmission of Chikungunya virus. Currently, there are no licensed antivirals to treat Chikungunya virus disease (CHIKVD). Even Chikungunya virus is not a life-threatening disease, it has a high morbidity impact in patients in an acute and long-term setting. In the future, the most effective way to control CHIKVD will include the use of a safe and effective vaccine. Three decades ago began significant progress in the development of a CHIKV vaccine with many promising efforts. 
Recently major vaccine approaches have been applied to CHIKV, but we need to find the most suitable vaccines. Current actions have been focused to develop a safe and effective vaccine. New modifications for the inactivated virus approach that look promising, to different scopes such as the novel strategies of recombinant vaccines seem to be highly effective in generating an effective immune response against the virus. ${ }^{43}$

\section{Recent status of Vaccine Progress against Chikungunya Virus}

Significant progress was achieved in the research and development of vaccinations against CHIK within past years. Additional vaccination strategies have been investigated with promising preclinical results including a chimeric alpha virusa DNA replicon vaccine encoding the CHIKV envelope, p62-E1 protein vaccines, GP E2 protein subunits, adenovirus-based vaccine and the recombinant modified vaccinia virus Ankara vaccine expressing CHIKV antigens (MVACHIKV). Only two candidates have currently qualified to enter phase II clinical trials, a Chikungunya virus-like particle-based vaccine and a recombinant live attenuated measles virusvectored vaccine. This review focuses on the recent status of vaccine progress against Chikungunya virus in humans and discusses the variety of immunization strategies, results of current human trials and promising vaccine candidates. ${ }^{6}$ The various advancement in the development of vaccines for the treatment of Chikungunyais shown in Figure 3.

Some vaccines developed for the treatment of Chikungunya acting at different stages of parasitic infection which are in different clinical trial phases are shown in Table $3 .^{43}$

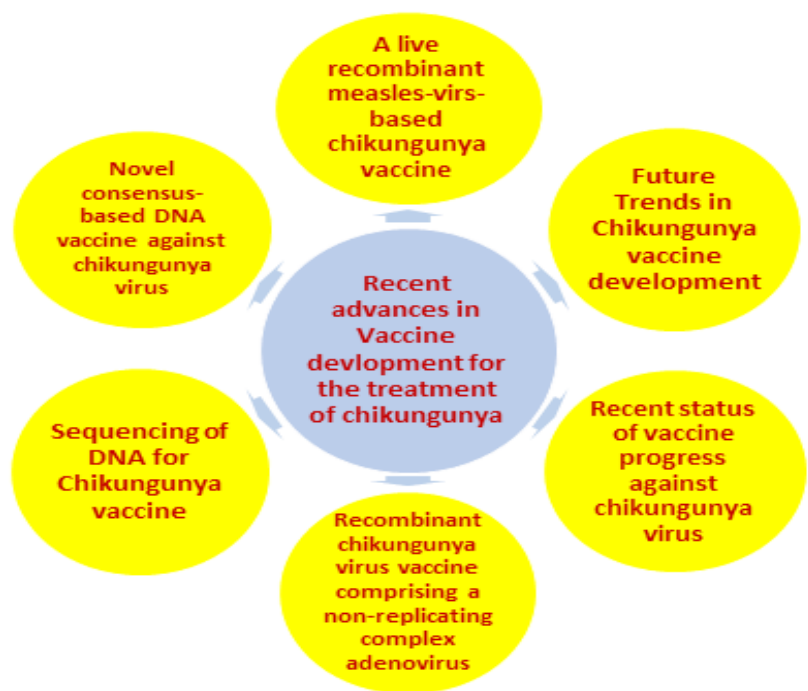

Figure 3: Advances in Vaccine Development for the Treatment of Chikungunya.

\section{Table 3: Current Status of Chikungunya vaccine Development.}

Current Prophylactic Chikungunya virus Vaccines

\begin{tabular}{|c|c|c|c|}
\hline Biological Components & Year & Phase & Ref. \\
\hline $\begin{array}{l}\text { Live Attenuated CHIKV181/25 } \\
\text { clone }\end{array}$ & 2000 & Phase II & 44 \\
\hline $\begin{array}{l}\text { Live Attenuated CHIKV/IRES } \\
\text { (version } 1 \text { and version 2) Pre- } \\
\text { clinical (Macaques) }\end{array}$ & 2014 & $\begin{array}{l}\text { Pre-clinical } \\
\text { (Macaques) }\end{array}$ & 45 \\
\hline $\begin{array}{l}\text { Live attenuated CHIV encoding } \\
\text { nsP3/6K }\end{array}$ & 2014 & $\begin{array}{l}\text { Pre-clinical } \\
\text { (Mice) }\end{array}$ & 46 \\
\hline Virus like particles & 2010 & $\begin{array}{l}\text { Pre-clinical } \\
\text { (Macaques) }\end{array}$ & 47 \\
\hline Virus like particles & 2014 & $\begin{array}{l}\text { Pre-clinical } \\
\text { (Macaques) }\end{array}$ & 48 \\
\hline $\begin{array}{l}\text { Adenovirus vector expressing: } \\
\text { envelope glycoproteins E1, E2 } \\
\text { and capsid (Cap) protein. }\end{array}$ & 2011 & $\begin{array}{l}\text { Pre-clinical } \\
\text { (Mice) }\end{array}$ & 40 \\
\hline $\begin{array}{l}\text { Recombinant measles virus } \\
\text { expressing: } \\
\text { CHIKV-like particles }\end{array}$ & 2013 & $\begin{array}{l}\text { Pre-clinical } \\
\quad \text { (Mice) }\end{array}$ & 49 \\
\hline $\begin{array}{l}\text { Chimeric vesicular stomatitis } \\
\text { virus expressing: } \\
\text { entire CHIKV envelope } \\
\text { polyprotein (E3-E2-6K-E1) }\end{array}$ & 2013 & $\begin{array}{l}\text { Pre-clinical } \\
\text { (Mice) }\end{array}$ & 50 \\
\hline $\begin{array}{l}\text { Recombinant modified vaccinia } \\
\text { virus Ankara expressing: } \\
\text { glycoprotein E2 of CHIKV }\end{array}$ & 2013 & $\begin{array}{l}\text { Pre-clinical } \\
\text { (Mice) }\end{array}$ & 50 \\
\hline $\begin{array}{c}\text { Recombinant modified vaccinia } \\
\text { virus Ankara expressing } C, E 3 \text {, } \\
\text { E2, } 6 K \text { and } E 1 \text { genes }\end{array}$ & 2014 & $\begin{array}{l}\text { Pre-clinical } \\
\text { (Mice) }\end{array}$ & 51 \\
\hline $\begin{array}{l}\text { DNA vaccine: cassette based } \\
\text { on } E 1, E 2 \text { and Cap }\end{array}$ & 2008 & $\begin{array}{l}\text { Pre-clinical } \\
\text { (Mice) }\end{array}$ & 52 \\
\hline
\end{tabular}

\section{CONCLUSION}

The emergence of drug resistance in malaria has led to the development of alternative approaches for the treatment of malaria and other similar infectious diseases like Dengue and Chikungunya. Some of the potent vaccines developed for malaria, such as RTS, S and Hepatitis $B$ virus surface antigen $(\mathrm{HBsAg})$ are in clinical phase trials. The vaccines for Dengue include the tetravalent vaccine. A live-attenuated tetravalent vaccine based on the chimeric yellow fever-Dengue virus (CYD-TDV) are being explored in clinical phase trials in many developed countries. Similarly iDNA plasmid pCHIKV-7 based 181/25 vaccine and particle-based vaccines have been developed for Chikungunya. After the successful outcomes of the developed vaccines for Malaria, Dengue and Chikungunya, a great victory is expected for the fight against these life-threatening diseases. 


\section{ACKNOWLEDGEMENT}

The authors wish to thank and acknowledge the support provided by the Management of Shivalik College of Pharmacy, Nangal (Punjab), G.D. Goenka University (Gurugram) and Baddi University of Emerging Sciences and Technology, Baddi, Himachal Pradesh.

\section{CONFLICT OF INTEREST}

The authors declare no conflict of interest.

\section{ABBREVIATIONS}

CHIKV: Chikungunya Virus; RNA: Ribonucleic Acid; WHO: World Health Organization; HBsAg: Hepatitis B Virus Surface Antigen; EPI: Expanded Program on Immunization; CYD: Chimeric Yellow Fever-Dengue Virus; TDV: Tetravalent Dengue Vaccine; DNA: Deoxyribonucleic Acid; GMP: Good Manufacturing Practices; YFV: Yellow Fever Vaccine; ELISA: Enzyme-linked Immune Sorbent Assay; WRAIR: Walter Reed Army Institute of Research; NIAID: National Institute of Allergy and Infectitous Diseases; NIH: National Institute of Health.

\section{REFERENCES}

1. World Health Organization (WHO). The World Health Report 1997. Conquering, suffering, enriching humanity. Geneva; World Health Organisation Publisher.

2. Vekemans J. Major global vaccine challenges: Recent progress in malaria vaccine development. The Vaccine Book. 2016;385-99.

3. Salazar-González JA, Angulo C, Rosales-Mendoza S. Chikungunya virus vaccines: Current strategies and prospects for developing plant-made vaccines. Vaccine. 2015;33(31):3650-8.

4. Smalley C, Erasmus JH, Chesson CB, Beasley DWC. Status of research and development of vaccines for Chikungunya. Vaccine. 2016;34(26):2976-81.

5. Weaver SC, Osorio JE, Livengood JA, Chen R, Weaver DTSW. Chikungunya virus and prospects for a vaccine. Expert Rev Vaccines. 2012;11(9):10871101

6. Schwameis M, Buchtele N, Wadowski PP, Choergenhofe C, Jilma B. Chikungunya vaccines in development. Human Vaccines and Immunotherapeutic. 2016;12(3):716-31.

7. Strauss JH, Strauss EG. The alpha viruses: Gene expression, replication and evolution. Microbiol Rev. 1994;58(3):491-562.

8. Chirathaworn C, Poovorawan Y, Lertmaharit S, Wuttirattanakowit N. Cytokine levels in patients with Chikungunya virus infection. Asian Pac J Trop Med. 2013;6(8):631.

9. Tang KF, Ooi EE. Diagnosis of dengue: An update. Expert Rev Anti Infect Ther. 2012;10(8):895-907.

10. World Health Organization. Dengue. Guidelines for diagnosis, treatment, prevention and control. 2017

11. Thomas SJ, Rothman AL. Trials and tribulations on the path to developing a Dengue vaccine. Vaccine. 2015;33(Suppl 4):24-31

12. Mustafa MMS, Agrawal CVK. Dengue vaccine: The current status. Med $\mathrm{J}$ Armed Forces India. 2008;64(2):161-4.

13. Crompton PD, Pierce SK, Miller LH. Advances and challenges in malaria vaccine development. J Clin Invest. 2010;120(12):4168-78.

14. Sauerwein RW, Bousema T. Transmission blocking malaria vaccines: Assays and candidates in clinical development. Vaccine. 2015;33(52):7476-82.
15. Draper SJ, Angov E, Horii T, Srinivasan P, Theisen M, Biswas S, et al. Recent advances in a recombinant protein-based malaria vaccine. Vaccine. 2015;33(52):7433-43

16. Birkett AJ. Status of vaccine research and development of vaccines for malaria. Vaccine. 2016;34(26):2915-20.

17. Satiric DI, Good MF. Whole organism blood stage vaccines against malaria. Vaccine. 2015;33(52):7469-75.

18. Hedstrom RC, Sedegah M, Hoffman SL. Prospects and strategies for the development of DNA vaccines against malaria. Res Immunol. 1994;145(6):476-90

19. Gilbert SC, Hill AVS. Protein particle vaccines against malaria. Parasitol Today. 1997;13(8):302-6

20. Galactionova K, Tediosi F, Camponovo F, Smith TK, Gething PW, Penny MA. Country-specific predictions of the cost-effectiveness of malaria vaccine RTS, S/AS01 in endemic Africa. Vaccine. 2017;35(1):53-60.

21. Abdulla S, Oberholzer R, Juma O, Kubhoja S, Machera F, Membi C, et al. Safety and immunogenicity of RTS, S/AS02D malaria vaccine in infants. N Engl J Med. 2008;359(24):2533-44

22. Goodman AL, Draper SJ. Blood-stage malaria vaccines: Recent progress and future challenges. Annals of Tropical Medicine and Parasitology. 2010;104(3):189-211.

23. Agnandji ST, Lell B, Fernandes JF, Abossolo BP, Methogo BG, Kabwende $A L$, et al. A Phase 3 trial of RTS, S/AS01 malaria vaccine in African infants. N Engl J Med. 2012;367(24):2284-95.

24. Malkin E, Dubovsky F, Moree M. Progress towards the development of malaria vaccines. Trends in Parasitology. 2006;22(7):7.

25. Reed ZH, Friede M, Kieny MP. Malaria vaccine development: Progress and challenges. Current Molecular Medicine. 2006;6(2):231-45.

26. McCarthy JS, Good MF. Whole parasite blood-stage malaria vaccines: A convergence of evidence. Human Vaccines. 2010;6(1):114-23.

27. Sinha S, Medhi K, Sehgal R. Challenges in drug resistant malaria. Parasite. 2014;21:61

28. Osorio JE, Partidos CD, Wallace D, Stinchcomb DT. Development of a recombinant, chimeric tetravalent dengue vaccine candidate. Vaccine. 2015;33(50):7112-20.

29. Ferguson NM, Rodríguez-Barraquer I, Dorigatti, I, Mier-Teran YRL, Laydon DJ, Cummings DA. Benefits and risks of the Sanofi-Pasteur dengue vaccine: Modeling optimal deployment. Science. 2016;353(6303):1033-6.

30. Rodriguez-Barraquer I, Mier-Teran YRL, Schwartz IB, Burke DS, Cummings DAT. Potential opportunities and perils of imperfect Dengue vaccines. Vaccine. 2014;32(4):514-20

31. Jindal $H$, Bhatt $B$, Malik JS. Dengue vaccine: A valuable asset for the future. Human Vaccine Immunother. 2014;10(8):2245-6.

32. Martin J, Hermida L. Dengue vaccine: An update on recombinant subunit strategies. Acta Virol. 2016;60(1):3-14.

33. Miller N. Recent progress in Dengue vaccine research and development Curr Opin Mol Ther. 2010;12(1):31-8.

34. Vannice KS, Roehrig JT, Hombach J. Next-generation Dengue vaccines: A review of the preclinical development pipeline. Vaccine. 2015;33(50):7091-9.

35. Guy B, Briand O, Lang J, Saville M, Jackson N. Development of the Sanofi Pasteur tetravalent dengue vaccine: one more step forward. Vaccine. 2015;33(50):7100-11.

36. Bhamarapravati N, Sutee Y. Live attenuated tetravalent dengue vaccine. Vaccine. 2000;18(Suppl 2):44-7.

37. Guy B, Saville M, Lang J. Development of Sanofi Pasteur tetravalent dengue vaccine. Human Vaccines. 2010;6(9):696-705

38. Lang J. Recent progress on Sanofi Pasteur's Dengue vaccine candidate. Journal of Clinical Virology. 2009;46(Suppl 2):20-4

39. Ramsauer K, Schwameis M, Firbas C, Mullner M, Putnak RJ, Thomas SJ, et al. Immunogenicity, safety and tolerability of are combinant measles-virusbased Chikungunya vaccine: A randomized, double-blind, placebo-controlled, active-comparator, first-in-man trial. Lancet Infect Dis. 2015;15(5):519-27.

40. Wang D, Suhrbier A, Penn-Nicholson A, Woraratanadharm J, Gardner $\mathrm{J}$, Luo $\mathrm{M}$, et al. A complex adenovirus vaccine against Chikungunya virus provides complete protection against viraemia and arthritis. Vaccine. 2011;29(15):2803-9. 
41. Muthumani K, Lankaraman KM, Laddy DJ, Sundaram SG, Chung CW, Sako $\mathrm{E}$, et al. immunogenicity of novel consensus-based DNA vaccines against Chikungunya virus. Vaccine. 2008;26(40):5128-34.

42. Hidajat R, Nickols B, Forrester N, Tretyakova I, Weaver S, Pushko P. Nextgeneration sequencing of DNA-launched Chikungunya vaccine virus. Virology. 2016;490:83-90.

43. Garcia A, Diego L, Judith B. New approaches to Chikungunya virus vaccine development. Recent Patents on Inflammation and Allergy Drug Discovery. 2015;9(1):31-7.

44. Edelman R, Tacket CO, Wasserman SS, Bodison SA, Perry JG, Mangiafico JA. Phase II safety and immunogenicity study of live Chikungunya virus vaccine TSI-GSD-218. Amer J Trop Med Hyg. 2000;62(6):681-5.

45. Roy CJ, Adams AP, Wang E, Plante K, Gorchakov R, Seymour RL. Chikungunya vaccine candidate is highly attenuated and protects nonhuman primates against telemetrically monitored disease following a single dose. J Infect Dis. 2014;209(12):1891-9.

46. Hallengärd D, Kakoulidou M, Lulla A, Kümmerer BM, Johansson DX, Mutso M. Novel attenuated Chikungunya vaccine candidates elicit protective immunity in C57BL/6 mice. J Virol. 2014;88(5):2858-66.
47. Akahata W, Yang ZY Andersen H, Sun S, Holdaway HA, Kong WP. A virus-like particle vaccine for epidemic Chikungunya virus protects nonhuman primates against infection. Nature Med. 2010;16(3):334-8.

48. Chang LJ, Dowd KA, Mendoza FH, Saunders JG, Sitar S, Plummer SH Safety and tolerability of Chikungunya virus-like particle vaccine in healthy adults: A Phase 1 dose-escalation trial. Lancet. 2014;384(9959):2046-52.

49. Brandler S, Ruffié C, Combredet C, Brault JB, Najburg V, Prevost MC. A recombinant measles vaccine expressing Chikungunya virus-like particles is strongly immunogenic and protects mice from lethal challenge with Chikungunya virus. Vaccine. 2013;31(36):3718-25.

50. Chattopadhyay A, Wang E, Seymour R, Weaver SC, Rose JK. Achimericvesiculo/alpha virus is an effective alpha virus vaccine. J Virol. 2013:87(1):395-402.

51. García-Arriaza J, Cepeda V, Hallengärd D, Sorzano CÓ, Kümmerer $\mathrm{BM}$, Liljestrom P. A novel poxvirus-based vaccine, MVA-CHIKV, is highly immunogenic and protects mice against Chikungunya infection. J Virol. 2014;88(6):3527-47.

52. Muthumani K, Lankaraman KM, Laddy DJ, Sundaram SG, Chung CW, Sako E. Immunogenicity of novel consensus-based DNA vaccines against Chikungunya virus. Vaccine. 2008:26(40):5128-34.

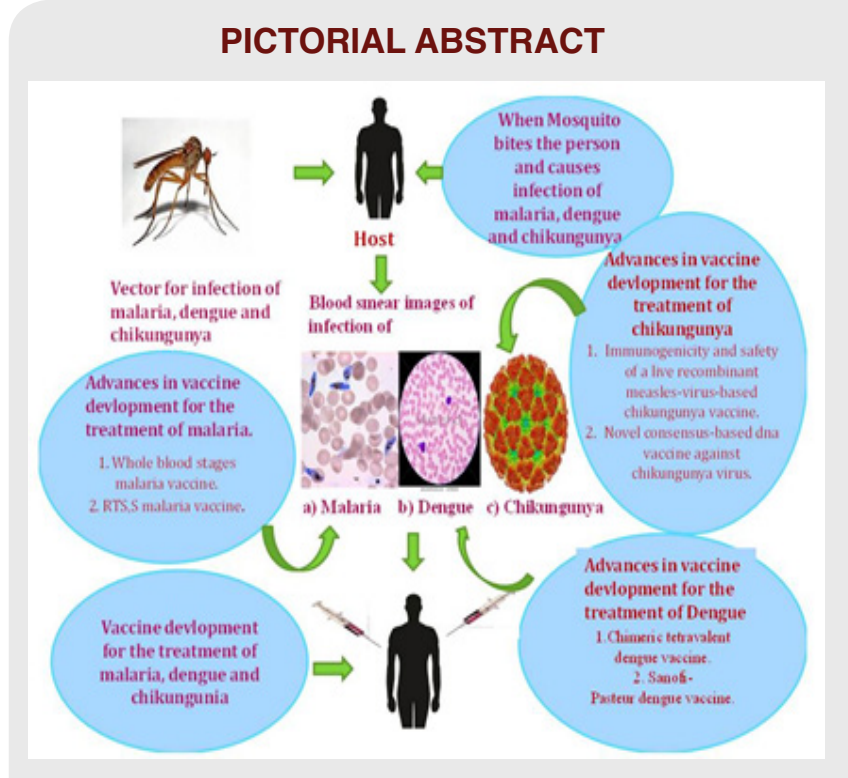

\section{ABOUT AUTHORS}

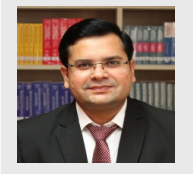

Dr. Sahil Kumar is working as an Assistant Professor at Department of Pharmacy, G.D. Goenka University, Gurgaon (Haryana), India. He has completed his B. Pharm. and M. Pharm from GJUST, Hisar (Haryana) and Ph.D. as Senior Research Fellow in DRDO, New Delhi funded research project at I.S.F. College of Pharmacy, Moga (Punjab). He has published more than 36 publications, one book, 08 best paper awards in many reputed National and International Journals and Conferences. He is gold medalist in Pharmaceutical Chemistry and recipient of DST-INSPIRE fellowship. He has guided 11 post graduate students and guiding $02 \mathrm{Ph} . \mathrm{D}$. students at present.

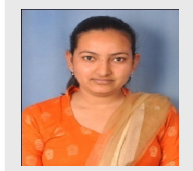

Kiran Thakur, (M. Pharmacy), School of Pharmacy and Emerging Sciences, Baddi University of Emerging Sciences and Technology, Baddi, Solan, India.

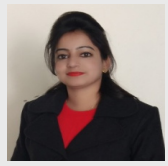

Bandna Sharma, (M. Pharmacy) is working as an Assistant Professor of Pharmaceutical Chemistry at School of Pharmacy and Emerging Sciences, Baddi University of Emerging Sciences and Technology, Baddi, Solan, India. 


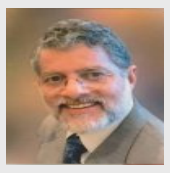

Prof. T.R. Bhardwaj is an Ex-Professor and Chairman, UIPS, Panjab University, Chandigarh and is currently working as Vice Chancellor and Dean, School of Pharmacy and Emerging Sciences, BUEST, Baddi, Himachal Pradesh. He has teaching and research experience of more than 40 years and published more than 150 publications in National and International Journals and Conferences and bagged several academic awards including Life Time Achievement Award in $69^{\text {th }}$ IPC-2017 at Chitkara University. He has invited as an eminent speaker for various FDP, Workshops and Conferences as resource persons. He has more than 15 International patents and guided several M. Pharm. and PhD students.

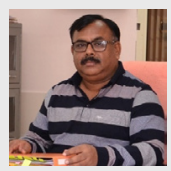

Dr. Deo Nandan Prasad, Ph.D., FIC is Principal and Professor, Shivalik College of Pharmacy. He received his B. Pharm (1985), M. Pharm (1987) and Ph.D. (2001) from Birla Institute of Technology, Ranchi, Jharkhand. He has teaching experience of more than 30 years and published more than 100 publications in National and International Journals and Conference. He has guided several M. Pharm. and $08 \mathrm{PhDs}$ and currently guiding $06 \mathrm{PhD}$ students. He is currently an Editorial Board Member of several journals, Ex-senator of Punjabi University, Patiala and Exchairman, Board of Studies, IKG Punjab Technical University, Jalandhar.

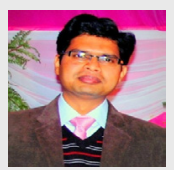

Dr. Rajesh Kumar Singh was born in 1980. He received his B. Pharm (2003) and M. Pharm (2005) from UIPS, Panjab University, Chandigarh and PhD from IKGPTU, Jalandhar in 2013. He has guided $17 \mathrm{M}$. Pharm. students and currently guiding $03 \mathrm{PhDs}$. He has to his credit more than 50 publications, 60 National and International Conference Abstracts, 2 Books and 6 Best Paper Awards. He is currently an Editorial Board Member of 08 SCOPUS indexed International Journals, reviewed more than 100 International papers and availed 1 Travel grant to attend the International Conference.

Cite this article: Kumar S, Thakur K, Sharma B, Bhardwaj TR, Prasad DN, Singh RK. Recent Advances in Vaccine Development for the Treatment of Emerging Infectious Diseases. Indian $\mathrm{J}$ of Pharmaceutical Education and Research. 2019;53(3):343-54. 